Marquette University

e-Publications@Marquette

College of Nursing Faculty Research and

Publications

Nursing, College of

$5-1-1990$

\title{
Methods Used to Self-Predict Ovulation A Comparative Study
}

Richard Fehring

Marquette University, richard.fehring@marquette.edu

Accepted version. Journal of Obstetric, Gynecologic, \& Neonatal Nursing, Vol. 19, No. 3 (May 1990): 233-237. DOI. (C) 1990 Blackwell Publishing. Used with permission. 


\section{Methods Used to Self-Predict Ovulation A Comparative Study}

Author: Richard J. Fehring, RN, DNSc

Abstract: Time of ovulation as detected by a self-test of luteinizing hormone $(\mathrm{LH})$ in the urine was compared with time of ovulation as detected by self-observation of cervical mucus. Twenty regularly cycling women monitored their cervical mucus and urine $L H$ for two complete menstrual cycles. Of the cycles that had an LH surge, $100 \%$ were on the peak day of cervical mucus or within three days before the peak day. Self-observation of cervical mucus, therefore, can be an accurate method of determining optimal fertility.

In recent years, a number of diagnostic self-test kits have been developed to help women detect their times of ovulation. ${ }^{1}$ The majority of these self-tests of ovulation are based on the detection of luteinizing hormone $(\mathrm{LH})$ in the urine. According to the World Health Organization, a surge in LH levels takes place about 16 hours before ovulation in the serum and about 6 hours later in the urine. ${ }^{2}$ The amount of LH in the serum or urine is detected by enzyme immunoassay of monoclonal antibodies. Urine LH self-test kits are used by women who want to time intercourse more precisely to become pregnant, often because they suffer from infertility problems. These ovulation detection self-test kits should not be used by women who wish to avoid pregnancy because such tests do not give an adequate warning time as to when ovulation is pending.

Peripheral changes in cervical mucus also should be good indirect indicators of ovulation. Estrogen levels peak about a day before ovulation, and estrogen stimulates the production of cervical mucus. A number of methods have been developed to help women detect their approximate times of ovulation through the self-observation of cervical mucus. One method, called the Creighton model, defines peak mucus as mucus that stretches an inch or more, is clear in appearance, and has the sensation of lubrication. ${ }^{3}$ The last day of one or more of these signs is considered the peak day. The peak day of cervical mucus has been shown by a number of researchers to correlate closely with the approximate time of ovulation and to be an inexpensive method of indirectly detecting ovulation. ${ }^{4-6}$

Some researchers argue that the variability of cervical mucus prevents the mucus from being an accurate means of detecting ovulation. ${ }^{7,8}$ Nulsen et al., however, compared the relationship of urinary LH detected by a self-test kit with maximum cervical mucus, as well as basal body temperature (BBT), of 10 spontaneously ovulating women. ${ }^{9}$ These researchers 1 Fehring 
found that BBT was not a good detector of ovulation and did not correlate well with the urinary LH surge. However, Nulsen et al. found that, in the majority of their research subjects, the day of maximum cervical mucus coincided closely with the day of the urine LH surge (i.g., 0-12 hours preceding the LH surge) and not 12-24 hours before or after the LH surge, as predicted by others. Maximum, or optimal, cervical mucus was determined by a physician using a mucus scoring system developed by Insler and others. ${ }^{10}$ The Insler scoring system is a quantitative measure based on the volume of cervical mucus, the stretch of the mucus, the degree of ferning that occurs, the amount of viscosity of the mucus, and the appearance of the cervix. ${ }^{10}$ The scoring system requires a physician to aspirate mucus directly from the cervix. Nulsen et al. modified the Insler scoring system by substituting the degree of cellularity of the cervical mucus for the appearance of the cervix. ${ }^{9}$ Using another laboratory rating system, Leader et al. also found optimal cervical mucus to be an accurate indicator of ovulation when compared to daily real-time ultrasonography. ${ }^{11}$

Although the Nulsen study showed that peak mucus symptoms correlated well with the time of the LH surge, ${ }^{9}$ the study was not a fair test in self-detection of ovulation in that the home ovulation urine test was compared with a laboratory determination of peak mucus. For the test to be fair, the women subjects should have determined their own optimal or peak mucus much as they determined their own LH surge through use of the self-test kit. Furthermore, the subjects in the study were undergoing infertility assessment and, therefore, were not ideal subjects because infertility patients, more than is likely with women of normal fertility, have certain conditions (e.g., anovulation, limited cervical mucus, or abnormal hormonal levels) that would interfere with the study results. Therefore, the purpose of this current research study was to compare the detected time of ovulation by a urine self-test of LH with the detected time of ovulation by a self-determination of the peak day of cervical mucus.

\section{Methodology}

The subjects for this study were 20 women between the ages of 22 and 41 years (mean, 29.4 years) who 1) had no known fertility problems, 2) had regular reproductive cycles, 3) did not smoke, and 4) were not on oral contraceptives or any other type of medication that affects the hormonal patterns or cervical mucus of the ovulation cycle. The 20 women were purposely selected from among clients who attended an academic nursing center's natural family-planning clinic. Each subject had been taught the Creighton model ovulation method and had completed a minimum of four follow-up teaching sessions with one of the nurses in the nursing center.

2 Fehring 
Each subject also was familiar with the Creighton model vaginal discharge recording system (VDRS) and had completed at least one cycle of cervical mucus charting.

The LH surges in the urine were self-detected by using a ovulation self-test kit based on enzyme immunoassay of the LH monoclonal antibodies in the urine. The test used has a reported LH sensitivity of $30 \mathrm{mIU} / \mathrm{ml}^{1}$ According to the manufacturer, the $\mathrm{LH}$ surge can be detected as early as 36 hours before ovulation with $98-100 \%$ accuracy. The reaction time of the enzymes with the antibodies in the urine is about six minutes with 10 drops of urine. Color changes are detected on a test pad.

The peak day of cervical mucus was determined by means of the Creighton model VDRS, developed through research conducted at St. Louis University and Creighton University over a five-year period. ${ }^{3}$ The recording system requires that women check for cervical mucus by wiping the .outside of their vaginas (the vulvar area) every time they go to the bathroom to void and once before going to bed. The women check the mucus for color, stretch, and consistency. The women then use the VDRS to rate and chart the mucus most likely to indicate fertility every day and the frequency of occurrence of this mucus. The VDRS has a reported $99 \%$ method effectiveness and a $95 \%$ use effectiveness for determining fertile and nonfertile days of the cycle., 12

After the subjects read and signed consent forms, they were each given two ovulation self-test kits and two fertility charts. Each test kit provided enough material for nine days of testing. The subjects were shown how to check their urine using the self-test kit according to the manufacturer's instructions. The subjects were asked to check their urine once a day with the kits between 10 A.M. and 8 P.M., at approximately the same time each day. The nine days of the subjects' cycles that the tests were to be used were determined by a chart provided in the manufacturer's instructions. The subjects were instructed to record positive signs on their fertility charts when they had positive changes on their test pads. The subjects also were instructed to continue to chart their menstrual cycles using the Creighton model and the VDRS.

\section{Results}

In the first cycle, 15 of the 20 subjects had detectable LH surges. Two of the subjects, both who were trying to achieve pregnancy, became pregnant during the first cycle. In the second cycle, 14 of the remaining 18 subjects had detectable LH surges. Therefore, a total of 29 of the 38 cycles had detectable LH surges (Table 1).

Of the 29 cycles that had LH surges, $9(31 \%)$ had the surges on the peak day, $9(31 \%)$

3 Fehring 
had the surges on the day before the peak day, $6(20.7 \%)$ had the surges two days before the peak day, 4 (13.8\%) had the surges three days before the peak day, and 1 cycle (3.5\%) with the LH surge did not have a peak day, Therefore, 28 (96.5\%) of the 29 cycles with LH surges had surges on or within three days before the peak day (Table 2).

\section{Discussion}

The results of this study are comparable to the studies by Nulsen et al. and Leader et al., which showed that cervical mucus is a good indicator of the approximate time of ovulation. ${ }^{9,11}$ However, unlike the subjects of those studies, the subjects in this study did daily observations of their own mucus and rated the mucus using the Creighton model VDRS. In the Nulsen and Leader studies, mucus was withdrawn from each subject's cervix by the researcher or an assistant with a syringe and rated with the Insler mucus scoring system. ${ }^{9,11}$

In the Nulsen study, eight subjects (57\%) had the maximum Insler score on the day of the urinary LH surge, and only one subject had a maximum score on the day after the LH surge. ${ }^{9}$ In the Leader study, $31 \%$ of the cycles had the maximum Insler score on the day of follicular rupture (as determined by ultrasound), and $47.5 \%$ of the cycles had the maximum score 24 hours before follicular rupture. ${ }^{11}$ In comparison, the study presented here had nine cycles (31\%) with the LH surge on the peak mucus day and nine cycles (31\%) with the LH surge on the day before the peak mucus day. No LH surges were observed on the day after the peak day.

One of the most interesting findings of the study is that only $77.7 \%$ of the cycles had a detectable LH surge, despite manufacturer claims of a greater than $90 \%$ accuracy in detecting the LH surge. This particular finding is surprising because the subjects were already knowledgeable about their reproductive cycles and had received detailed written instructions and demonstrations of the use of the ovulation self-testing kits. This lower rate of LH detection, however, is consistent with the results of the once-a-day testing conducted in the Nulsen study. ${ }^{9}$

In the Nulsen study, 9 of 10 cycles had detected LH surges. ${ }^{9}$ However, if morning-only testing had been used, only 6 of 10 cycles would have detected LH surges and if afternoon-only testing were used, just 7 of 10 cycles would have detected LH surges. Both the Nulsen study and the study presented here used urinary LH test kits manufactured by the same company, but the current study cycles used a newer and faster version.

The reasons that once-a-day testing was used for this study were that 1) the manufacturer's instructions indicate using the test only once a day, 2) the expense of using the 4 Fehring 
test more than once a day would be prohibitive, and 3) the testing time for the subjects would be greater (doubled) if the tests were used more than once a day. The intent of the study was to use the LH test kits as they would be used in actual clinical practice.

Six of the nine cycles that did not have detectable LH surges were either 25 days or shorter or 35 days long. This could mean that even if twice-a-day testing were used, the LH surge could have been missed because of the variable lengths of the cycles (early or late ovulation). The problem could be alleviated by having the subjects test for the LH surge on a daily basis through the entire cycle; however, this would be very expensive, and the self-test kits only include nine days of testing material. All but one of the cycles had a detectable peak day. However, this does not mean that all of those cycles were ovulatory.

\section{Nursing Implications}

The most important finding of this study is that self-observation of cervical mucus, through the use of the Creighton model ovulation method, is a highly accurate way to determine the approximate time of optimal fertility. In this study, 28 (96.5\%) of the 29 of the LH surges were detected on the peak day (the last day of peak cervical mucus) or within three days before the peak day. Because ovulation appears to occur about 12-48 hours after the LH surge, and since sperm will survive about three days in good mucus, the peak mucus day or the day after the peak mucus day in all of the cycles with LH surges were excellent days to have intercourse if the subjects were trying to achieve pregnancy. The peak days in the cycles that did not have LH surges also might have been optimal fertile days if ovulation occurred. The reason that the peak days in the LH surge cycles that went undetected might have been optimal fertile days is that, from the results of both the current study and the Nulsen study, one would expect the LH surge to be detected in only $60-78 \%$ of cycles when once-a-day testing is used, even if all of the cycles were ovulatory.

The implication of the $\mathrm{LH}$ detection kit rate is that a nurse might recommend to a woman who is using the kit that, to achieve almost $100 \%$ certainty in detecting an LH surge, twice-a-day testing should be used. Testing also should occur during the major proportion of the menstrual cycle (i.e., from the tail end of menses until an LH surge is detected). However, the subject also must be made aware that continuous twice-a-day testing will be expensive and time consuming. Most of the subjects in this study indicated that self-observation of their cervical mucus was more convenient and less time consuming than testing their urine for the LH surge. Also, nurses should realize that self-observation of cervical mucus is inexpensive and provides a woman with

\section{Fehring}


a host of information about her menstrual cycle. Daily self-observation of cervical mucus will give information about the length of the menstrual cycle; the approximate length of the luteal phase; the nature and length of the menstrual period; whether or not any unusual bleeding has occurred; whether or not any cervical or vaginal inflammation is present; and the nature, quality, and amount of the cervical mucus. For example, the second cycle of subject 18 (Table 1) did not have any observable cervical mucus. Therefore, even though this subject probably ovulated (as indicated by the LH surge), she would have difficulty getting pregnant because of the dry cycle. If she were using just the LH detection kit, she would have no knowledge of this infertility problem.

One criticism of the mucus method is that the method does not work when a woman has continuous mucus caused by cervical or vaginal inflammation or infections, or when seminal fluid is present. However, although determining fertility or infertility is more difficult when continuous or extraneous mucus is present, a woman can be taught techniques by a qualified natural family-planning practitioner (NFPP) to help her identify her fertile time with confidence. Seminal fluid is managed simply by having the woman expel the fluid from her vagina using Kegel exercises and bearing down exercises within an hour after intercourse. If a continuous mucus discharge is present, the woman is taught how to distinguish an "essentially the same" mucus pattern from her mucus cycle. The important nursing implication of all of this is that those women who learn how to identify their optimal fertile times through daily observation of cervical mucus need to be referred to a nurse certified in natural family planning (NFP) or to a NFPP. Furthermore, referral should be made only to a nurse or NFPP who uses a well-researched and well-developed educational delivery model (e.g., the Creighton model). A directory of NFPPs and nurse NFPPs who use the Creighton model can be obtained from the Pope Paul VI Reproductive Institute in Omaha, Nebraska.

\section{Conclusion}

Accurately predicting and timing of ovulation are important steps in assessing and treating female infertility. Predicting ovulation also is critical for the millions of couples who rely on abstinence for family planning. Daily self-observation of cervical mucus, when taught by a qualified NFPP, can be an accurate and inexpensive means of determining optimal days of fertility.

\section{Notes}

6 Fehring 
- This research study was funded by a grant from Sigma Theta Tau International.

- Address for correspondence: Richard J. Fehring, RN, DNSc, Associate Professor, Marquette University, College of Nursing, 510 North 16th Street, Milwaukee, Wisconsin 53233.

- Richard J. Fehring. RN. DNSc. is an associate professor at the Marquette University College of Nursing and the coordinator of the Marquette University Natural Family Planning Services. Dr. Fehring is a member of NAACOG and Sigma Theta Tau and is president-elect of the American Academy of Natural Family Planning.

\section{References}

1. Batzer, F. 1986. Test kits for ovulation and pregnancy. Technology 1987: Contemporary OB/GYN. 28:7-16.

2. World Health Organization. 1980. Task force on methods for the determination of the fertile period. Am J Obstet Gynecol. 138:383-90.

3. Hilgers, T.W., D. Daly, S. Hilgers, and A. Prebil. 1982. The Ovulation Method of NFP. Omaha: Creighton University NFP Center.

4. Hilgers, T.W., G.E. Abraham, and D. Cavanaugh. 1978. Natural family planning I: The peak symptom and estimated time of ovulation. Obstet Gynecol. 52:575-82.

5. Moghissi, K. 1980. Prediction and detection of ovulation. Fertil Steril. 34:89-98.

6. Moghissi, K. 1986. Cervical mucus changes and ovulation prediction and detection. J Reprod Med. 31 (Supplement):7 48-53.

7. Seibel, M. 1986. Luteinizing hormone and ovulation timing. J Reprod Med. 31(Supplement):754-59.

8. Corson, SL. 1986. Panel discussion. J Reprod Med. 31(Supplement):770-72.

9. Nulsen, J., C. Wheeler, M. Ausmanas, and L. Blasco. 1987. Cervical mucus changes in relationship to urinary luteinizing hormone. Fertil Steril. 48:783-86.

10. . Insler, V., H. Melamed, I. Eichenbrenner, D. Seer, and B. Lunenfeldt. 1982. The cervical score: A simple semiquantitative method for monitoring of the menstrual cycle. Int $\mathrm{J}$ Fertil. 10:223-28.

11. Leader, A., D. Wiseman, and P.J. Taylor. 1985. The prediction of ovulation: A comparison of the basal body temperature graph, cervical mucus score, and real time pelvic ultrasonography. Fertil Steril. 43:385-88.

12. . Doud, J. 1985. Use-effectiveness of the Creighton model of NFP. International

7 Fehring 
Review of Natural Family Planning. 9:54-72.

\section{Appendix}

Table 1

Comparison of LH Surge with Peak Mucus Day

\begin{tabular}{|c|c|c|c|c|c|c|}
\hline Subject & Age (years) & P/G & LH-1 & Peak-1 & LH-2 & Peak-2 \\
\hline 1 & 34 & $5 / 4$ & 16 & 16 & 14 & 15 \\
\hline 2 & 23 & $0 / 0$ & 15,16 & 19 & 14,15 & 16 \\
\hline 3 & 41 & $0 / 0$ & 11 & 12 & 12 & 14 \\
\hline 4 & 38 & $0 / 0$ & 14 & 15 & 15 & 15 \\
\hline 5 & 38 & $4 / 4$ & - & 15 & - & 14 \\
\hline 6 & 24 & $0 / 0$ & 17 & 19 & 14,15 & 15 \\
\hline 7 & 29 & $1 / 1$ & 20 & 21 & Pregnant & \\
\hline 8 & 24 & $1 / 1$ & - & 12 & 16 & 16 \\
\hline 9 & 30 & $1 / 1$ & - & 17 & 12,13 & 13 \\
\hline 10 & 28 & $2 / 2$ & 13,14 & 15 & 13,14 & 17 \\
\hline 11 & 28 & $1 / 1$ & - & 14 & - & 17 \\
\hline 12 & 22 & $0 / 0$ & 17 & 18 & - & 15 \\
\hline 13 & 34 & $5 / 3$ & 16 & 18 & Pregnant & \\
\hline 14 & 27 & $1 / 1$ & 16 & 17 & 16 & 16 \\
\hline 15 & 29 & $0 / 0$ & 17,18 & 18 & - & 17 \\
\hline 16 & 24 & $0 / 0$ & 17,18 & 20 & 16,17 & 20 \\
\hline 17 & 26 & $0 / 0$ & - & 18 & 14,15 & 18 \\
\hline 18 & 37 & $1 / 0$ & 15 & 16 & 16 & - \\
\hline 19 & 27 & $0 / 0$ & 15 & 17 & 15 & 17 \\
\hline 20 & 25 & $0 / 0$ & 16 & 16 & 15 & 16 \\
\hline
\end{tabular}

$\mathrm{P} / \mathrm{G}=$ Para/Gravida.

LH-1; Peak-1; LH-2; Peak-2 = day of the LH surge and peak mucus day of the first and second menstrual cycle, respectively.

8 Fehring 


\section{Table 2}

Relationship of Peak Mucus Day to LH Surge

\begin{tabular}{|l|c|c|c|c|c|c|}
\hline & $\mathbf{P}-\mathbf{4}$ & $\mathbf{P}-\mathbf{3}$ & $\mathbf{P}-\mathbf{2}$ & $\mathbf{P}-\mathbf{1}$ & $\mathbf{P}$ & $\mathbf{P}+\mathbf{1}$ \\
\hline Number of LH surges & 0 & 4 & 6 & 9 & 9 & 0 \\
\hline Percent of LH surges & 0 & 13.8 & 20.7 & 31 & 31 & 0 \\
\hline
\end{tabular}

$\mathrm{P}=$ the peak day of cervical mucus. The minus sign (-) indicates days before $\mathrm{P}$ and the plus sign (+) indicates says after. 